\title{
Lung cancer masquerading as atrial myxoma
}

\author{
AH AL-HILLAWI, R HAYWARD, N McI JOHNSON, S PAYNE, T TREASURE
}

From the Middlesex Hospital, London

Case report

A 53 year old Greek seaman and lifelong heavy smoker (60 cigarettes a day) was referred for treatment of a suspected bronchial carcinoma. He had noticed some palpita- ' tions in the preceding weeks and a chest radiograph showed a mass $8 \mathrm{~cm}$ in diameter within the apical segment of the left lower lobe. The day before he attended he had noted headache, difficulty with vision, and left sided paraesthesiae.

On examination he was in sinus rhythm and had an abnormal cardiac impulse, which was tapping in quality. There was a loud first heart sound but no opening snap. A mid-diastolic murmur was heard intermittently. He had left homonymous hemianopia. Carcinoma of the bronchus with cerebral secondaries was diagnosed clinically.

Fibreoptic bronchoscopy showed an obstruction in the apical segment of the left lower lobe but a biopsy was inconclusive. Percutaneous fine needle aspiration biopsy confirmed the diagnosis of malignancy, the cell type being uncertain. Computerised tomography showed an abnormality in the right posterior cerebral cortex which had the appearances of infarction rather than secondary tumour. In view of the abnormal heart sounds the possibility of embolism from the heart was considered and echocardiography was performed to exclude coexistent mitral stenosis. Two dimensional echocardiography showed an intracardiac mass with the appearances of a left atrial myxoma.

Although the possibility that this was an intracardiac extension of the bronchial carcinoma was considered the appearances were thought suggestive of myxoma. As there had already been one cerebral infarct urgent surgery was performed to prevent further embolism.

At operation via a median sternotomy bypass was established and the left atrium was opened through the usual right sided incision parallel to the atrioventricular groove. A $20 \mathrm{~cm}$ semilobulated tongue of tissue (fig) passing from the left atrium through the mitral valve was discovered. An intra-atrial attachment was sought but the tumour was seen to be protruding from the left inferior pulmonary vein. This vessel was opened from the outside and the tumour, together with the mass in the apical segment of the left lower lobe, was removed from the left side of the heart. It was removed complete with the left lung. Histological examination of the tumour showed it to be an anaplastic bronchial carcinoma.

Address for correspondence: Dr AH Al-Hillawi, Department of Medicine, Middlesex Hospital, London W1N SAA.

Accepted 3 May 1983

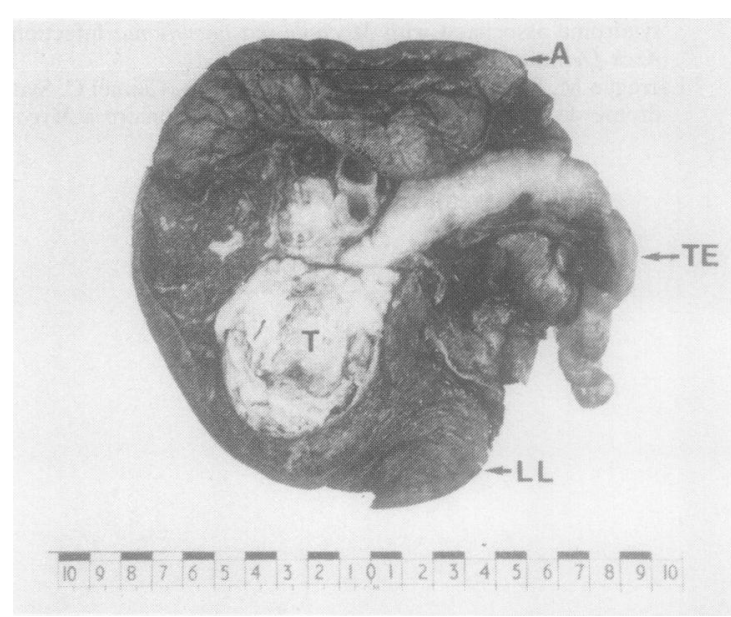

Medial surface of the left lung. A-apex; LL-lower lobe; $T$-tumour; TE-tumour extension.

The patient made a good recovery and left hospital with normal heart sounds and no neurological signs.

\section{Discussion}

In this case the occurrence of cerebral embolism and the confusing findings on auscultation, suggestive but not typical of mitral stenosis, were characteristic of the presentation of left atrial myxoma. Furthermore, the echocardiographic findings were considered to support this diagnosis. While lung cancers may affect the left atrium, such a large intracardiac extension of tumour lying free within the pulmonary vein is uncommon. It has been reported at necropsy when widespread metastases have occurred. ${ }^{12}$ We have found no record of a similar presentation of lung cancer or of its successful surgical removal.

Cerebral embolism has been described in non-bacterial 9 thrombotic endocarditis due to underlying lung carcinoma, ${ }^{3}$ but there was no evidence of tumour invasion of the heart or vegetations of the valves in this case. Whether cerebral embolism was due to the tumour itself or to $N$ thrombus or platelets from its surface remains uncertain; the resolution of the neurological signs suggests the latter.

\section{References}

${ }^{1}$ Hanfling SM. Metastatic cancer to the heart. Circulation 1960; 22:474.

${ }^{2}$ Rogen AS, Moffatt AD. Unusual secondary tumour of the heart. Br Heart J 1967; 29:638-40.

${ }^{3}$ Studdy P, Willoughby JMT. Non-bacterial thrombotic endocarditis in early cancer. $\mathrm{Br} M e d J$ 1976; i:752. 